\title{
BLIND PHASE NOISE ESTIMATION IN OFDM SYSTEMS BY SEQUENTIAL MONTE CARLO METHOD
}

\author{
Erdal Panayırc1 \\ Bilkent University \\ Department of Electrical and Electronics Engineering \\ Bilkent 06800, Ankara, Turkey
}

Hakan A. Çırpan

Istanbul University

Department of Electrical-Electronics Engineering

Avcilar 34850, Istanbul, Turkey

Marc Moeneclaey and Nele Noels

Ghent University

TELIN/DIGCOM Department

B9000 Gent, Belgium

\begin{abstract}
In this paper, based on a sequential Monte Carlo method, a computationally efficient algorithm is presented for estimating the residual phase noise, blindly, generated at the output the phase tracking loop employed in OFDM systems. The basic idea is to treat the transmitted symbols as "missing data" and draw samples sequentially of them based on the observed signal samples up to time $t$. This way, the Bayesian estimates of the phase noise is obtained through these samples, sequentially drawn,together with their importance weights. The proposed receiver structure is seen to be ideally suited for high-speed parallel implementation using VLSI technology.
\end{abstract}

\section{Introduction}

One of the main drawbacks of OFDM systems is the phase noise (PN) caused by the oscillator instabilities [1]. Unfortunately, due to the PN, the most valuable feature namely orthogonality between the carriers, is destroyed resulting in a significant degradation of the performance 
of OFDM systems [1]. Random PN causes two effects on OFDM systems, rotating each symbol by a random phase that is referred to as the common phase error (CPE) and producing intercarrier interference (ICI) term that adds to the channel noise due to the lost of orthogonality between subcarriers [2]. Several methods have been proposed in the literature for the estimation and compensation of the PN in OFDM systems $[3,4]$. Most of the approaches however only addresses the estimation of CPE by assuming ICI terms is approximated by a Gaussian distribution and these techniques are implemented after the DFT process at the receiver [4]. The main drawback of these approaches is the data dependent ICI which introduces an additional random noise on top of the additive Gaussian channel noise causes a significant degradation in the CPE estimator performance. In contrast to these approaches, we try to solve PN estimation problem in the time domain before the DFT process at the OFDM receiver. As it will be seen next section this approach will not be faced with ICI effect during the estimation procedure resulting in more accurate random phase estimation. The method proposed is based on the sequential monte Carlo techniques. The basic idea is to treat the transmitted symbols as "missing data" and to sequentially draw samples of them based on the current observation and computing appropriate importance sampling weights. Based on sequentially drawn samples, the Kalman filter is used to estimate the unknown phase from a extended Kalman state-space model of the underlying system. Furthermore, the tracking the time-varying PN and the data detection are naturally integrated. The algorithm is self-adaptive and no training/pilot symbols or decision feedback are needed.

\section{System Description}

We consider an OFDM system with $N$ subcarriers operating over a frequency selective Rayleigh fading channel. In this paper we assume that the multipath intensity profile has exponential distribution and the delay spread $T_{d}$ is less than or equal to the guard interval $L$. With the aid of the discrete time channel model [6], the output of the frequency selective channel can be written as $y_{t}=\sum_{k=0}^{L} h_{k} s_{t-k}$ where the $h_{k}, k=0,1, \ldots, L$ denotes the $k$ th tap gain and we assume to have ideal knowledge of these channel tap gains. Also, assuming perfect frequency and timing synchronization, the received signal, $r_{t}$, corrupted by the additive Gaussian noise $n_{t}$ and distorted by the time-varying phase noise $\theta_{t}$ can be expressed as

$$
r_{t}=y_{t} e^{j \theta_{t}}+n_{t}, \quad t=1, \cdots T_{0}
$$


where $s_{t}=\sum_{n=0}^{N-1} d_{n} e^{-j \frac{2 \pi t n}{N}}$. Here $\left\{d_{n}\right\}$ denotes the independent data symbols transmitted on the $n$th subcarrier of an OFDM symbol. We assume that $d_{n}$ 's are M-PSK symbols taking values in the set $\left\{e^{-j \frac{2 \pi r}{M}}, r=\right.$ $0,1, \cdots, M-1\}$. Hence, $s_{t}$ is a linear combination of independent, identically distributed random variables. If the number of subcarriers is sufficiently large, $s_{t}$ can be modelled a a complex Gaussian process whose real and imaginary parts are independent. It has zero mean and variance $\sigma_{s}^{2}=E\left\{\left|s_{t}\right|^{2}\right\}=E_{s}$, where $E_{s}$ is the symbol energy per subcarrier. $n_{t}$ is the complex envelope of the additive white Gaussian noise with variance $\sigma_{n}^{2}=E\left\{\left|n_{t}(k)\right|^{2}\right\}$. $\theta_{t}$ is the sample of the PN process at the output of the free-running local oscillator representing the phase noise. It can be shown that PN can be modelled as a Wiener process defined as

$$
\theta_{t}=\theta_{t-1}+u_{t} \quad \text { where } \quad \theta_{0} \sim \text { uniform }(-\pi, \pi)
$$

where $u_{t}$ is zero-mean Gaussian random variable with variance $\sigma_{u}^{2}=$ $2 \pi B T_{s}$ where $T_{s}$ is the sampling period of the OFDM receiver $\mathrm{A} / \mathrm{D}$ converter and $B T$ refers to the $\mathrm{PN}$ rate, where $T=T_{s}(N+L)$. It is assumed that $u_{t}$ and $n_{t}$ are independent of each other. Defining the vectors $\boldsymbol{R}_{t}=\left[r_{0}, r_{1}, \cdots, r_{t}\right]^{T}, \boldsymbol{S}_{t}=\left[\boldsymbol{s}_{0}, \boldsymbol{s}_{1}, \cdots \boldsymbol{s}_{t}\right]^{T}, \boldsymbol{s}_{t}=\left[s_{t}, s_{t-1}, \cdots s_{t-L}\right]^{T}$, and $\boldsymbol{h}_{t}=\left[h_{0}, h_{1}, \cdots, h_{L}\right]^{T}$, combining (1), (2) and taking into account the structure of $s_{t}$, we obtain the following dynamic state-space representation of the communication system,

$$
\theta_{t}=\theta_{t-1}+u_{t}, \quad \boldsymbol{s}_{t}=\boldsymbol{F} \boldsymbol{s}_{t-1}+\boldsymbol{v}_{t}, \quad r_{t}=\boldsymbol{h}^{T} \boldsymbol{s}_{t} e^{i \theta_{t}}+n_{t}
$$

where

$$
\boldsymbol{F}=\left[\begin{array}{cccc}
0 & 0 & \cdots & 0 \\
0 & 1 & \cdots & 0 \\
. & . & \cdots & . \\
0 & 0 & \cdots & 1
\end{array}\right]
$$

is a $(L+1) \times(L+1)$ shifting matrix, and $\boldsymbol{v}_{t}=\left[s_{t}, 0, \cdots, 0\right]$ is a $(L+1) \times 1$ perturbation vector that contains the new symbol $s_{t}$.

Since we are interested in estimating the the phase noise $\theta_{t}$ blindly at time $t$ based on the observation $\boldsymbol{R}_{t}$, the Bayes solution requires the posterior distribution

$$
p\left(\theta_{t} \mid \boldsymbol{R}_{t}\right)=\int p\left(\theta_{t} \mid \boldsymbol{R}_{t}, \boldsymbol{S}_{t}\right) p\left(\boldsymbol{S}_{t} \mid \boldsymbol{R}_{t}\right) d \boldsymbol{S}_{t} .
$$

Note that with a given $\boldsymbol{S}_{t}$, the nonlinear (Kalman filter) model (3) can be converted into a linear model by linearizing the observation equation (1) as follows [7]:

$$
\theta_{t}=\theta_{t-1}+u_{t}, \quad \text { and } \quad r_{t}=\boldsymbol{h}^{T} \boldsymbol{s}_{t}\left(V_{t} \theta_{t}+Q_{t}\right)+n_{t}
$$


where $V_{t}=j e^{j \widehat{\theta}_{t \mid t-1}}$ and $Q_{t}=\left(1-j \widehat{\theta}_{t \mid t-1}\right) e^{j \widehat{\theta}_{t \mid t-1}} . \widehat{\theta}_{t \mid t-1}$ denotes the estimator of $\theta_{t}$ based on the observations $\boldsymbol{R}_{t-1}=\left(r_{0}, r_{1} \cdots, r_{t-1}\right)$. Then the state-space model (3) becomes a linear Gaussian system. Hence, $p\left(\theta_{t} \mid \boldsymbol{S}_{t}, \boldsymbol{R}_{t}\right) \sim N\left(\mu_{\theta_{t}}\left(\boldsymbol{S}_{t}\right), \sigma_{\theta_{t}}^{2}\left(\boldsymbol{S}_{t}\right)\right)$, where the mean $\mu_{\theta_{t}}\left(\boldsymbol{S}_{t}\right)$ and the variance $\sigma_{\theta_{t}}^{2}\left(\boldsymbol{S}_{t}\right)$ can be obtained as follows. Denoting $\mu_{\theta_{t}}\left(\boldsymbol{S}_{t}\right) \triangleq \widehat{\theta}_{t \mid t}$, and $\sigma_{\theta_{t}}^{2}\left(\boldsymbol{S}_{t}\right) \triangleq M_{t \mid t}$.

$\widehat{\theta}_{t \mid t}$ and $M_{t \mid t}$ can be calculated recursively by using the Extended Kalman Technique [ [7], page 449-452] with the given $\boldsymbol{S}_{t}$ as:

$$
\begin{aligned}
\widehat{\theta}_{t \mid t} & =\widehat{\theta}_{t \mid t-1}+K_{t}\left(r_{t}-\boldsymbol{h}^{T} \boldsymbol{s}_{t} e^{j \widehat{\theta}_{t \mid t-1}}\right) \\
M_{t \mid t} & =\left(1-K_{t} V_{t} M_{t \mid t-1}\right)
\end{aligned}
$$

where

$$
K_{t}=\frac{M_{t \mid t-1} V_{t}^{*}}{\left(M_{t \mid t-1}+\sigma_{n}^{2}\right)}, \quad \widehat{\theta}_{t \mid t-1}=\widehat{\theta}_{t-1 \mid t-1}, \quad M_{t \mid t-1}=M_{t-1 \mid t-1}+\sigma_{u}^{2} .
$$

\subsection{SMC for Blind Phase Noise Estimation}

We can now make timely estimates of $\theta_{t}$ based on the currently available observation $\boldsymbol{R}_{t}$, up to time $t$, blindly, as follows. With the Bayes theorem, we realize that the optimal solution to this problem is

$$
\widehat{\theta}_{t}=E\left\{\theta_{t} \mid \boldsymbol{R}_{t}\right\}=\int_{\boldsymbol{S}_{t}} \underbrace{\left[\int_{\theta_{t}} \theta_{t} p\left(\theta_{t} \mid \boldsymbol{S}_{t}, \boldsymbol{R}_{t}\right) d \theta_{t}\right]}_{\mu_{\theta_{t}}\left(\boldsymbol{S}_{t}\right)} p\left(\boldsymbol{S}_{t} \mid \boldsymbol{R}_{t}\right), d \boldsymbol{S}_{t} .
$$

In most cases, an exact evaluations of the expectation (9) is analytically intractable. Sequential Monte Carlo technique can provide us an alternative way for the required computation. Specifically, following the notation adopted in [8], if we can draw $m$ independent random samples $\left\{\boldsymbol{S}_{t}^{(j)}\right\}_{j=1}^{m}$ from the distribution $p\left(\boldsymbol{S}_{t} \mid \boldsymbol{R}_{t}\right)$, then we can approximate the quantity of interest $E\left\{\theta_{t} \mid \boldsymbol{R}_{t}\right\}$ in (9) by $E\left\{\theta t \mid \boldsymbol{R}_{t}\right\} \cong \frac{1}{m} \sum_{j=1}^{m} \mu_{\theta_{t}}\left(\boldsymbol{S}_{t}^{(j)}\right)$. But, usually drawing samples from $p\left(\boldsymbol{S}_{t} \mid \boldsymbol{R}_{t}\right)$ directly is usually difficult. Instead, sample generation from some trial distribution may be easier. In this case, the idea of importance sampling can be used [8]. By associating the weight $w_{t}^{(j)}=\frac{p\left(\boldsymbol{S}_{t}^{(j)} \mid \boldsymbol{R}_{t}\right)}{q\left(\boldsymbol{S}_{t}^{(j)} \mid \boldsymbol{R}_{t}\right)}$ to the samples, the quantity of interest, $E\left\{\theta_{t} \mid \boldsymbol{S}_{t}\right\}$ can be approximated as follows:

$$
E\left\{\theta_{t} \mid \boldsymbol{R}_{t}\right\} \cong \frac{1}{W_{t}} \sum_{j=1}^{m} \mu_{t}\left(\boldsymbol{S}_{t}^{(j)}\right) w_{t}^{(j)}
$$


with $W_{t}=\sum w_{t}^{(j)}$. The pair $\left(\boldsymbol{S}_{t}^{(j)}, w_{t}^{(j)}\right), j=1,2, \ldots, m$ is called a properly weighted sample with respect to distribution $p\left(\boldsymbol{S}_{t} \mid \boldsymbol{R}_{t}\right)$.

Specifically, it was shown in [8] that a suitable choice for the trial distribution is of the form $q\left(s_{t} \mid \boldsymbol{R}_{t}, \boldsymbol{S}_{t-1}^{(j)}\right)=p\left(s_{t} \mid \boldsymbol{R}_{t}, \boldsymbol{S}_{t-1}^{(j)}\right)$. For this trial sampling distribution, it is shown in [8] that the importance weight is updated according to

$$
w_{t}^{(j)}=w_{t-1}^{(j)} p\left(r_{t} \mid \boldsymbol{R}_{t-1}, \boldsymbol{S}_{t-1}^{(j)}\right), \quad t=1,2, \cdots
$$

The optimal trial distribution in (11) can be computed as follows:

$$
p\left(s_{t} \mid \boldsymbol{R}_{t}, \boldsymbol{S}_{t-1}^{(j)}\right)=p\left(r_{t} \mid \boldsymbol{R}_{t-1}, \boldsymbol{S}_{t-1}^{(j)}, s_{t}\right) P\left(s_{t} \mid \boldsymbol{R}_{t-1}, \boldsymbol{S}_{t-1}^{(j)}\right)
$$

Furthermore, it can be shown from the state and observation equations in (3) that $p\left(r_{t} \mid \boldsymbol{R}_{t-1}, \boldsymbol{S}_{t-1}^{(j)}, s_{t}\right) \sim \mathcal{N}\left(\mu_{r_{t}}^{(j)}, \sigma_{r_{t}}^{2(j)}\right)$ with mean and variance given by

$$
\begin{aligned}
\mu_{r_{t}}^{(j)} & =E\left\{r_{t} \mid \boldsymbol{R}_{t-1}, \boldsymbol{S}_{t-1}^{(j)}, s_{t}\right\}=\boldsymbol{h}^{T} \boldsymbol{s}_{t}\left(V_{t} \widehat{\theta}_{t \mid t-1}^{(j)}+Q_{t}\right) \\
\sigma_{r_{t}}^{2(j)} & =\operatorname{Var}\left\{r_{t} \mid \boldsymbol{R}_{t-1}, \boldsymbol{S}_{t-1}^{(j)}, s_{t}\right\}=\left|\boldsymbol{h}^{T} \boldsymbol{s}_{t}\right|^{2} M_{t \mid t-1}^{(j)}+\sigma_{n}^{2}
\end{aligned}
$$

where the quantities $\widehat{\theta}_{t \mid t-1}^{(j)}$ and $M_{t \mid t-1}^{(j)}$ in (13) can be computed recursively for the Extended Kalman equations given in (7), (8). Also since $s_{t}$ is independent of $\boldsymbol{S}_{t-1}$ and $\boldsymbol{R}_{t-1}$, the second term in (12) can be written as $p\left(s_{t} \mid \boldsymbol{R}_{t-1}, \boldsymbol{S}_{t-1}^{(j)}\right)=p\left(s_{t}\right)$ where it was pointed out earlier that $p\left(s_{t}\right) \sim \mathcal{N}\left(0, \sigma_{s}^{2}\right)$.

Note that dependency of the $\sigma_{r_{t}}^{2(j)}$ in (13) to $s_{t}$ precludes combining the product of Gaussian densities in (12) into a single Gaussian, hence obtaining a tractable sampling distribution. This problem can be circumvented by approximating the $\sigma_{r_{t}}^{2(j)}$ as follows. From (3), we can use the approximation $\boldsymbol{s}_{t} \approx \boldsymbol{F} \boldsymbol{s}_{t-1}$ in (13) to obtain

$$
\sigma_{r_{t}}^{2(j)} \cong\left|\boldsymbol{h}^{T} \boldsymbol{F} \boldsymbol{s}_{t-1}^{(j)}\right|^{2} M_{t \mid t-1}^{(j)}+\sigma_{n}^{2}
$$

Similarly using (9) in (13), the mean $\mu_{r_{t}}^{(j)}$ can be expressed as $\mu_{r_{t}}^{(j)}=$ $\left(\boldsymbol{h}^{T} \boldsymbol{F} \boldsymbol{s}_{t-1}^{(j)}+h_{0} s_{t}\right) G_{t}^{(j)}$ where $G_{t}^{(j)} \triangleq V_{t} \widehat{\theta}_{t \mid t-1}^{(j)}+Q_{t}$. Then, the true trial sampling distribution $p\left(s_{t} \mid \boldsymbol{R}_{t}, \boldsymbol{S}_{t-1}^{(j)}\right)$ in (12) can be expressed as follow:

$$
p\left(s_{t} \mid \boldsymbol{R}_{t}, \boldsymbol{S}_{t-1}^{(j)}\right) \sim \mathcal{N}\left(\mu_{s_{t}}^{(j)}, \sigma_{s_{t}}^{2(j)}\right)
$$


where

$$
\begin{aligned}
\mu_{s_{t}}^{(j)} & =\frac{\left(r_{t}-\boldsymbol{h}^{T} \boldsymbol{F} \boldsymbol{s}_{t-1}^{(j)} G_{t}^{(j)}\right)}{h_{0} G_{t}^{(j)}}\left(\frac{\sigma_{r_{t}}^{2(j)}}{\left|h_{0} G_{t}^{(j)}\right|^{2} \sigma_{s}^{2}}+1\right)^{-1} \\
\sigma_{s_{t}}^{2(j)} & =\frac{\sigma_{r_{t}}^{2(j)} \sigma_{s}^{2}}{\sigma_{r_{t}}^{2(j)}+\left|h_{0} s_{t} G_{t}^{(j)}\right|^{2} \sigma_{s}^{2}}
\end{aligned}
$$

and $\sigma_{r_{t}}^{2(j)}$ is defined as (13).

In order to obtain the recursion for the weighting factor $w_{t}^{(j)}$, the predictive distribution $p\left(r_{t} \mid \boldsymbol{R}_{t-1}, \boldsymbol{S}_{t-1}^{(j)}\right)$ in (12) should be evaluated. It is given by

$$
\begin{aligned}
p\left(r_{t} \mid \boldsymbol{R}_{t-1}, \boldsymbol{S}_{t-1}^{(j)}\right) & =\int_{s_{t}} p\left(r_{t} \mid \boldsymbol{R}_{t-1}, \boldsymbol{S}_{t-1}^{(j)}, s_{t}\right) p\left(s_{t} \mid \boldsymbol{R}_{t-1}, \boldsymbol{S}_{t-1}^{(j)}\right) d s_{t} \\
& =\int_{s_{t}} p\left(r_{t} \mid \boldsymbol{R}_{t-1}, \boldsymbol{S}_{t-1}^{(j)}, s_{t}\right) p\left(s_{t}\right) d s_{t}
\end{aligned}
$$

where (15) holds because $s_{t}$ is independent of $\boldsymbol{S}_{t-1}$ and $\boldsymbol{R}_{t-1}$. Since the the both terms in the integrand of (15), are Gaussian densities, the product of the Gaussian densities are integrated with respect to $s_{t}$ is also Gaussian. Therefore the predictive distribution is found to be

$$
p\left(r_{t} \mid \boldsymbol{R}_{t-1}, \boldsymbol{S}_{t-1}^{(j)}\right) \sim \mathcal{N}\left(M_{r_{t}}^{(j)}, \Sigma_{r_{t}}^{2(j)}\right),
$$

where $M_{r_{t}}^{(j)}=\boldsymbol{h}^{T} \boldsymbol{F} \boldsymbol{s}_{t-1}^{(j)} G_{t}^{(j)}$ and $\Sigma_{r_{t}}^{2(j)}=\left|h_{0} G_{t}^{(j)}\right|^{2} \sigma_{s_{t}}^{2}+\left|\boldsymbol{h}^{T} \boldsymbol{F} \boldsymbol{s}_{t-1}^{(j)}\right|^{2} M_{t \mid t-1}^{(j}+$ $\sigma_{n}^{2}$. We now summarize the SMC blind data phase noise estimation algorithm in Table I:

TABLE I

SMC ALGORITHM FOR BLIND PHASE NOISE ESTIMATION

Given $\left\{h_{0}, h_{1}, \cdots, h_{L}\right\}$

- Initialization:

- Initialize the extended Kalman filter: Choose the initial mean and the variance of the estimated $\theta_{t}$ as

$$
\mu_{\theta_{0}}^{(j)}=\widehat{\theta}_{0 \mid 0}^{(j)}=0, \quad \sigma_{\theta_{0}}^{2(j)}=M_{0 \mid 0}^{(j)}=\pi^{2} / 12, \quad j=1,2, \cdots, m .
$$

- Initialize the importance weights: All importance weights are initialized as $w_{0}^{(j)}=1, j=1,2, \cdots, m$.

For $j=1, m$

For $t=1, T_{0}$

Given $\widehat{\theta}_{t \mid t-1}, M_{t \mid t-1}^{(j)}, \boldsymbol{S}_{t-1}^{(j)}$ 
- Compute $\mu_{r_{t}}^{(j)}, \sigma_{r_{t}}^{2(j)}$ from equations (13).

- Compute sampling distribution mean/variance $\mu_{s_{t}}^{(j)}, \sigma_{s_{t}}^{2(j)}$ from the equation (15).

- Sample $s_{t}^{(j)} \sim N\left(\mu_{s_{t}}^{(j)}, \sigma_{s t}^{(j)}\right)$ and Append $s_{t}^{(j)}$ to $\boldsymbol{S}_{t-1}^{(j)}$ to obtain $\boldsymbol{S}_{t}^{(j)}=\left(s_{t}^{(j)}, \boldsymbol{S}_{t-1}^{(j)}\right)$.

- Compute the importance weights:

$$
w_{t}^{(j)}=w_{t-1}^{(j)} p\left(r_{t} \mid \boldsymbol{R}_{t-1}, \boldsymbol{S}_{t-1}^{(j)}\right),
$$

where $p\left(r_{t} \mid \boldsymbol{R}_{t-1}, \boldsymbol{S}_{t-1}^{(j)}\right)$ is computed from equation (17)

- Compute Kalman updates $\widehat{\theta}_{t \mid t-1}^{(j)}, M_{t \mid t-1}^{(j)}$ from equations (7).

- Update the a posteriori mean and variance of the phase noise: If the samples drawn up to time $t$ is $\boldsymbol{S}_{t}$, set

$$
\begin{aligned}
\mu_{\theta_{t}}\left(\boldsymbol{S}_{t}^{(j)}\right) & \triangleq \mu_{\theta_{t}}^{(j)}=\widehat{\theta}_{t \mid t}^{(j)} \\
\sigma_{\theta_{t}}^{2(j)}\left(\boldsymbol{S}_{t}^{(j)}\right) & \triangleq \sigma_{\theta_{t}}^{2(j)}=M_{t \mid t}^{(j)} \quad j=1,2, \cdots, m .
\end{aligned}
$$

and update according to the Kalman equations (8).

- Do the re-sampling as described in [8]. next $\mathrm{j}$

- Estimate phase noise $\widehat{\theta}_{t}=\frac{1}{m} \sum_{j=1}^{m} \mu_{\theta_{t}}\left(\boldsymbol{S}_{t}^{(j)}\right)$

next $\mathrm{t}$

\section{Simulation Results}

In this section, we provide some computer simulation examples to demonstrate the performance of the proposed SMC approach for blind phase noise estimation and data detection in OFDM systems. The phase process is modelled by AR process driven by a white Gaussian noise with $\sigma_{u}^{2}=0.001 . s_{t}$ is modelled as a complex Gaussian process which has zero mean and variance $\sigma_{s}^{2}=1$. In order to demonstrate the performance of the adaptive SMC approach, we present the tracking performance for both phase and symbols at $S N R=20 d B$ in Fig. 1. It is shown through simulations that the performance of the proposed SMC algorithm can track the phase as well as transmitted symbols close to the true values.

\section{Conclusions}

We have developed a new adaptive Bayesian approach for blind phase noise estimation and data detection for OFDM systems based on sequential monte carlo methodology. The optimal solutions to joint symbol detection and phase noise estimation problem is computationally prohibitive to implement by conventional methods. Thus the proposed sequential approach offers an novel and powerful approach to tackling this problem at a reasonable computational cost. 

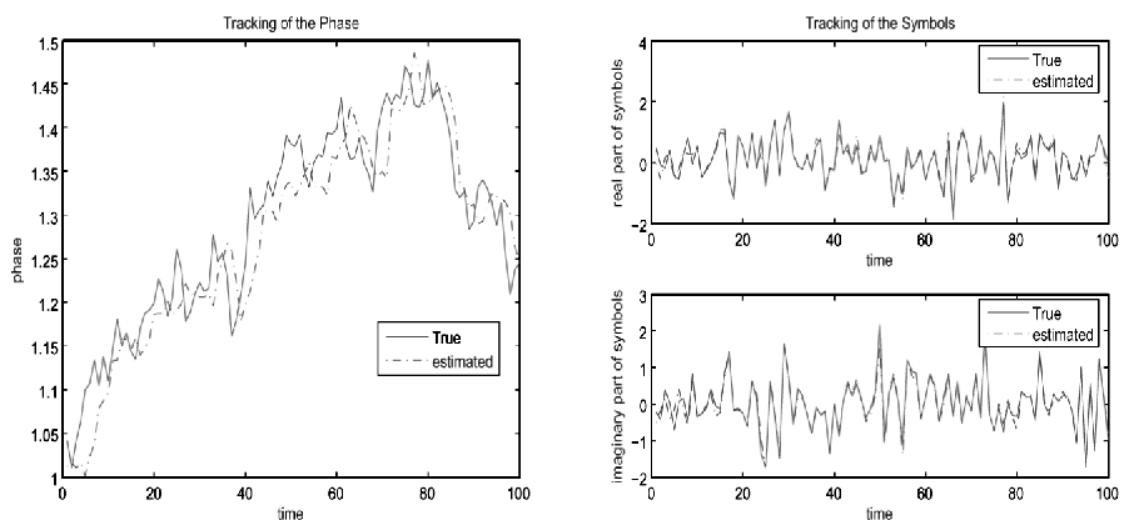

Figure 1. Tracking Performance

\section{Acknowledgments}

This paper has been produced as part of the NEWCOM Network of Excellence, a project funded by the European Commission's 6th Framework Programme. This work was also supported in part by the Research Fund of the University of Istanbul. Project number: UDP$599 / 28072005,220 / 29042004$.

\section{References}

[1] M.V.B.T. Pollet and M. Moeneclaey, "BER sensitivity of OFDM systems to carrier frequency offset and wiener phase noise," In IEICE Transactions on Communications, vol. 43, Feb/Mar/Apr 1995, pp. 192-193.

[2] H. Meyr, M. Moeneclaey and S.A. Fechtel, Digital Communications Receivers, John Wiley, New York, 1998.

[3] S. Wu and Y. Bar-Ness, "A Phase Noise Suppression Algorithm for OFDM-Based WLANs," IEEE Communications Letters vol. 44, no. 3, May 1998.

[4] D. Petrovic, W. Rave, and G. Fetweis, "Phase Noise Suppression in OFDM Including Intercarrier Interference" In Proc. Intl. OFDM Workshop, 2003.

[5] M. Pitt and N. Shephard, "Filtering via simulation: auxiliry particle filter", J. Amer. Statist. Soc. B, no. 63, 2001.

[6] J.J. van de Beek, O. Edfors, M. Sandell, S.K. Wilson, and P.O. Börjesson, "On channel estimation in OFDM systems," In Proc. 45th IEEE Vehicular Technology Conf.(VTC'96), Atlanta, GA, April 1996.

[7] S.M. Kay, "Fundamentals of Statistical Signal Processing: Estimation Theory," Prentice Hall 1993.

[8] Z. Yang and X. Wang, "A sequential Monte Carlo blind receiver for OFDM systems in frequency-selective fading channels", IEEE Trans. Signal Proc.,vol. 50, no. 2, pp. 271-280, Feb. 2003. 